\title{
Association of cytotoxin production and neutrophil activation by strains of Helicobacter pylori isolated from patients with peptic ulceration and chronic gastritis
}

\author{
Q B Zhang, I M Nakshabendi, M S Mokhashi, J B Dawodu, C G Gemmell, R I Russell
}

\begin{abstract}
Background-Helicobacter pylori is associated with neutrophil infiltration within the gastroduodenal mucosa. Neutrophil activation provides a major source of oxygen free radicals, which have been implicated in the pathogenesis of peptic ulceration.
\end{abstract}

Aim-To investigate if cytotoxin producing strains of $\boldsymbol{H}$ pylori are associated with the generation of oxidative burst in polymorphonuclear neutrophils (PMNs).

Patients-76 patients undergoing endoscopy of whom 45 had peptic ulcer and 31 chronic gastritis only were studied.

Methods-Strains of $\boldsymbol{H}$ pylori were cultured in Brucella broth. After 48 hours, bacteria were harvested by centrifugation and a bacterial suspension prepared as a stimulus for PMN oxidative burst using chemiluminescence. PMNs were prepared from healthy blood donors. To test the ability of strains to produce cytotoxin, culture supernatants of each were concentrated by polyethylene glycol and tested on cultured Vero cells for intracellular vacuolation.

Results-30 of $45(66 \cdot 7 \%)$ peptic ulcer patients induced cell vacuolation versus nine of $31(29 \%)$ strains from patients with chronic gastritis only $(\mathbf{p}<0 \cdot 01)$. Cytotoxin positive strains of $\boldsymbol{H}$ pylori regardless of the presence or absence of peptic ulcer displayed an increased induction of respiratory burst in PMNs compared with toxin negative strains from patients with chronic gastritis only $(p<0.05)$. Among the toxin negative strains, those from patients with peptic ulcer did not show a significant increase of the oxidative burst than those from patients without peptic ulcer (NS). Conclusion-Toxinogenicity of strains of $H$ pylori seems to be correlated with neutrophil respiratory burst and peptic ulceration. The ability of some strains of $H$ pylori to produce cytotoxin and to induce the oxidative burst in neutrophils may be important in the pathogenesis of peptic ulcer disease.

(Gut 1996; 38: 841-845)

Keywords: Helicobacter pylori, cytotoxin, free oxygen radicals, peptic ulcer.

The mechanisms by which Helicobacter pylori causes gastroduodenal mucosal damage are not well established. Free oxygen radicals have been implicated in a wide spectrum of human disease, ${ }^{1}$ including gastrointestinal disorders ${ }^{2} 3$ and may play an important part in gastroduodenal inflammation and peptic ulcer. ${ }^{4}$ A prominent histological feature of $H$ pylori infection is a dense infiltration of polymorphonuclear cells in the epithelium and the underlying lamina propria, which could be an important source of free radicals. ${ }^{56}$ Once activated, the neutrophils release free oxygen radicals, which may damage mucosal integrity. ${ }^{7}$ There is evidence to show that $H$ pylori stimulates gastric antral mucosal reactive oxygen radical production in vivo. ${ }^{8}$

Several potential bacterial virulence factors of $H$ pylori may contribute to gastroduodenal mucosal damage, such as cytotoxin and urease. ${ }^{10}$ Recent studies suggest that cytotoxin is associated with peptic ulceration and chronic active gastric inflammation, as is the CagA protein, which is strongly associated with cytotoxicity. ${ }^{1112}$

A previous report ${ }^{13}$ showed that $H$ pylori surface proteins that contain urease could activate blood monocytes, leading to the secretion of inflammatory cytokines and reactive oxygen intermediates, although the protein(s) that serve as the monocyte activator is not known. Another study shows that there is a protein with an estimated molecular weight of $150 \mathrm{kDa}$, which is associated with neutrophil activation. ${ }^{14}$

In this study, we have investigated if a relation exists between cytotoxin producing strains of $H$ pylori and the generation of free oxygen radicals by neutrophils and their relation to peptic ulcer disease.

\section{Methods}

\section{Patients}

Seventy six patients undergoing endoscopy in Glasgow Royal Infirmary were recruited in this study. These consisted of 45 with peptic ulcer ( 36 duodenal ulcer, nine gastric ulcer), and 31 with chronic gastritis only. Forty four patients were male (age between 20-75, mean 57), and 32 were female (age 24-73, mean 55). At endoscopy, gastric biopsy specimens were taken for culture, CLO test (Delta West Ltd, Australia), and histological examination. This study was approved by the Glasgow Royal Infirmary University of Glasgow NHS Trust Ethics Committee.
Correspondence to: Dr Q B Zhang, Departmen of Gastroenterology, Royal Infirmary,

Accepted for publication 13 December 1995 
Isolation of $\mathrm{H}$ pylori

Seventy six clinical isolates of $H$ pylori were studied. All the strains were positive for urease, oxidase, catalase, and showed typical morphology under light microscopy. For cytotoxin measurement and chemiluminescence assay, the strains were cultured in Brucella broth (Unipath, Basingstoke) containing $5 \%$ fetal bovine serum (Sigma, UK) at $37^{\circ} \mathrm{C}$ under microaerophilic conditions using a BBL Campypak (Cokeysville, USA), on a rotary shaker $(150 \mathrm{rpm})$. After 48 hours, the broth cultures were examined under microscopy for the typical morphology of curved bacilli and the percentage of coccoid formed strains in each culture was recorded. The percentage was found to be similar for all the strains in this study, which was $18-20 \%$. The cultures were then centrifuged at $3000 \mathrm{rpm}$ for 15 minutes, bacteria collected for chemiluminescence assay, and culture supernatants for cytotoxin assay. A broth culture without the inoculation of $H$ pylor $i$ was also prepared in the same way.

\section{Cytotoxin assay}

Culture supernatants of $H$ pylori were collected and filtered through $0.4 \mu \mathrm{m}$ filters (Millipore, France). The culture supernatants were then transferred to dialysis tubing (Sigma, UK) capable of retaining molecules with molecular weight greater than $12 \mathrm{kDa}$ and concentrated 20 -fold by reverse dialysis versus polyethylene glycol (Sigma, UK) as described earlier. ${ }^{15}$ Cultured Vero cells in Earle's medium (Gibco, Paisley) were used to measure intracellular vacuolation attributable to the cytotoxin according to the methods of Cover et al. ${ }^{16}$ Each concentrated culture supernatant was diluted from 1:5 to 1:160 and incubated for 24 hours with Vero cells in 96 well tissue culture plates. Cell vacuolation was quantified both visually and spectrophotometrically by following the uptake of neutral red. The maximum dilution of supernatant that produced vacuolation was defined as the titre of cytotoxin. During the assay, the broth culture supernatant without the inoculation of $H$ pylori was used as a toxin negative control.

\section{Chemiluminescence assay}

Fresh cultures of $H$ pylori were washed three times with saline. A bacterial suspension was prepared and adjusted to a concentration of $5 \times 10^{7} / \mathrm{ml}$. Healthy young adults without a history of peptic ulcer and under no treatment were used as white cell donors. Venous blood was separated by density gradient centrifugation (Polymorphprep, Nycomed, Birmingham, UK) and the polymorphonuclear leucocytes were washed twice in gel-Hanks's solution and standardised to contain $1 \times 10^{7} / \mathrm{ml}$. Free oxygen radical formation by polymorphonuclear neutrophils (PMNs) in response to $H$ pylori was studied by luminol dependent chemiluminescence assay. Seventy six nonopsonised strains and 42 strains opsonised with $10 \%$ normal human serum were tested in parallel in the test. The oxidative burst of
PMNs was recorded in cps (count per second) in a Luminometer (Canberra Packard, Caversham, UK). The peak value (cps) and the time (min) to reach the peak value were recorded for each strain. The assay for each sample was counted at three to four minute intervals for at least 60 minutes and all the bacterial suspensions were tested three times.

\section{Statistical methods}

The $\chi^{2}$ test and Student's $t$ test were used for two group comparisons. A two way analysis of variance and multiple comparison procedure was used to determine the independent effect of toxinogenicity and the presence or absence of peptic ulcer on the chemiluminescence response induced by strains of $H$ pylori.

\section{Results}

\section{Production of cytotoxin}

Concentrated culture supernatants from 39 of $76(51.3 \%)$ of the isolates of $H$ pylori produced measurable cell vacuolation. Among the strains from peptic ulcer patients 30 of 45 $(66.7 \%)$ induced cell vacuolation versus nine of $31(29 \%)$ strains from patients with chronic gastritis only $(p<0.01)$ (Table I). Some variation in toxinogenicity of the various strains was seen with titres of toxin ranging from 0 to 160 . The mean titre of cytotoxin produced by the strains from patients with peptic ulcer was 22.67 versus 7.74 in patients with chronic gastritis only $(p<0 \cdot 01,95 \%$ CI $4 \cdot 96,24 \cdot 9)$.

\section{Generation of reactive oxygen radicals}

The Figure shows the chemiluminescence response induced by 76 non-opsonised strains of $H$ pylori. It shows that there is strain to strain variation in the ability to induce the oxidative burst in PMNs displayed by the difference in the peak count (kcps) and the time to reach peak ( $\min )$. Some produced a stronger and more rapid response than others. The chemiluminescence pattern for each strain was reproducible. Although there are some small variations in peak height for each strain on each test occasion, the peak time was fairly constant (Table II).

\section{Reactive oxygen radicals, cytotoxin production,} and the presence of peptic ulcer disease

Using a two way analysis of variance approach, the effect of toxin status and the presence or absence of peptic ulcer on each strain's ability

TABLE I Cytotoxin production by strains of $\mathrm{H}$ pylori isolated from 76 patients with either peptic ulcer or chronic gastritis only

\begin{tabular}{lll}
\hline \multirow{2}{*}{$\begin{array}{l}\text { Toxinogenicity } \\
\text { of strains }\end{array}$} & \multicolumn{2}{l}{ Prevalence among patients with } \\
${$\cline { 2 - 3 }$} }$ & Chronic gastritis only \\
\hline Tox+ & $30 / 45^{\star}$ & $9 / 31$ \\
Tox- & $15 / 45$ & $22 / 31$ \\
\hline
\end{tabular}

${ }^{\star} \mathrm{p}<0.01$, compared with patients with chronic gastritis only $\left(\chi^{2}\right.$ test $)$. 


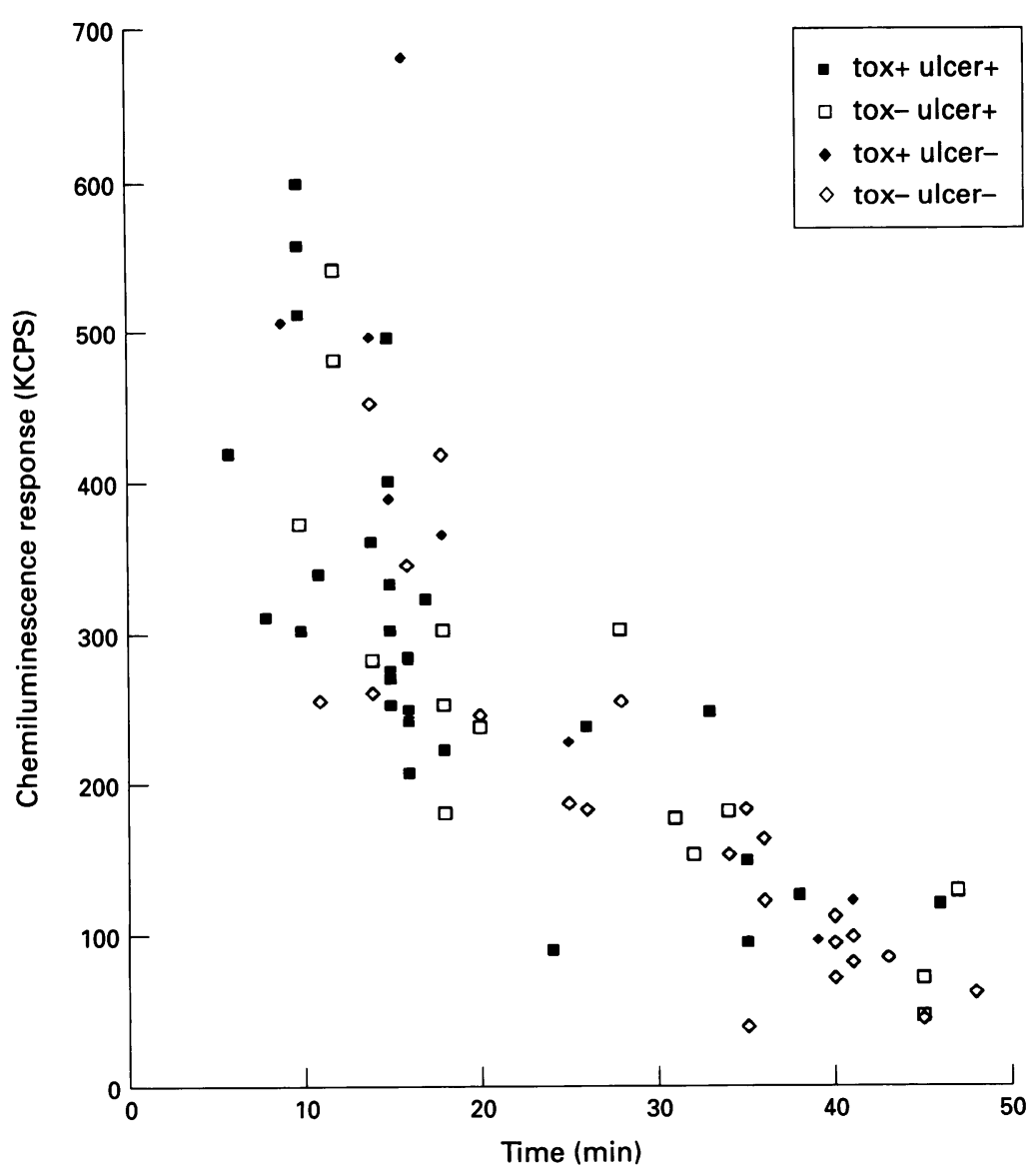

Neutrophil respiratory burst (as peak count of chemiluminescence) with 76 clinical isolates of $\mathrm{H}$ pylori. Results are the mean of three separate experiments and shown as (kilocount per second, kcps) and the time to reach peak (min).

to induce a chemiluminescence response in terms of peak count and the time to reach peak were investigated. Table III summarises and shows that the chemiluminescence response in terms of peak count induced by toxin positive strains regardless of the presence or absence of peptic ulcer (groups 1 and 2) is higher than that induced by those toxin negative strains from patients without peptic ulcer (group 4) $(p<0.05)$. It also shows that toxin positive strains (groups 1 and 2) take less time to induce peak chemiluminescence than those strains of group $4(p<0.05)$. These results show that the toxinogenicity of $H$ pylori may have a significant effect on the chemiluminescence response, although there is no significant difference between toxin positive strains (groups 1 and 2) and group 3, which were toxin negative but associated with peptic ulceration $(p>0.05)$. However, among the

TABLE II Chemiluminescence response induced by two representative strains of $\mathrm{H}$ pylori in three experiments. Strain $5617 B$ induced $a$ higher and quicker chemiluminescence response. $7994 \mathrm{~K}$ induced a weaker and slower one

\begin{tabular}{llll}
\hline Strains & Test & Peak value (kcps) & Peak time (min) \\
\hline 5617B & 1 & 534 & 15 \\
& 2 & 490 & 15 \\
& 3 & 458 & 16 \\
$7994 \mathrm{~K}$ & 1 & $494(38 \cdot 2)^{\star}$ & 39 \\
& 2 & 918 & 40 \\
& 3 & 71 & 40 \\
& & $93(23 \cdot 6)^{\star}$ & \\
\hline
\end{tabular}

^Mean (SD). toxin negative strains, those from patients with peptic ulcer failed to show any significant enhancement of the chemiluminescence response compared with those from patients without peptic ulcer (group 4) $(p>0.05)$ in terms of peak count and the time to reach peak.

When opsonised with $10 \%$ human serum, most strains of $H$ pylori resulted in a stronger and more rapid chemiluminescence response than non-opsonised, whether or not the test serum contained any $\mathrm{H}$ pylori antibody. A few of (seven of 42) strains that induced a high chemiluminescence response when non-opsonised gave a slightly decreased peak count (mean (SD) decrease: $65.4(36.5)$ ), while the time to reach peak was relatively unchanged. As can be seen from Table IV, there is no significant difference between the four groups of strains ( $p>0.05)$, indicating that the toxin status and the presence or absence of an ulcer do not affect the chemiluminescence response induced by opsonised strains. The presence of $H$ pylori antibody in any sera used for opsonisation did not affect the results.

\section{Discussion}

From our study, $66 \cdot 7 \%$ non-opsonised strains of $H$ pylori from peptic ulcer patients induced cell vacuolation, which is significantly higher than those from patients without confirmed peptic ulcer $(29 \%)$. This supports a previous report that the cytotoxin producing strains were more frequently found in patients with peptic ulcer. ${ }^{11}$ The mean titre of toxin produced in vitro was also higher in those strains from peptic ulcer patients than that from patients with chronic gastritis only ( 22.7 versus $7 \cdot 7$ ). Using a two way analysis of variance approach, we investigated the effect of toxinogenicity and the presence or absence of peptic ulcer on the oxidative burst induced by nonopsonised strains of $H$ pylori. It is found that cytotoxin producing strains have an increased chemiluminescence response in terms of higher peak count and shorter time to reach peak than those toxin negative strains from patients without peptic ulcer. These results support a possible link between the neutrophil respiratory burst and the toxinogenicity of strains of $H$ pylori. The association of certain strains with peptic ulcer disease in itself did not influence significantly the chemiluminescence response of human PMN. However, this finding does not exclude a possible relation between the neutrophil respiratory burst and peptic ulceration, as most strains from peptic ulcer patients $(66.7 \%$ in this study) are cytotoxin producing. Also we cannot rule out the possibility that some chronic gastritis patients infected with a cytotoxin producing strain might have had an ulcer in the past or may develop one in the future.

Most opsonised strains of $H$ pylori gave an increased chemiluminescence response in our study and the serum antibody to $H$ pylori showed no effect on the chemiluminescence response. This is consistent with previous reports, ${ }^{17} 18$ which suggested that the serum 
TABLE III Peak count and time to reach peak in the chemiluminescence response induced by 76 strains of $\mathrm{H}$ pylori in relation to the toxinogenicity and the presence or absence of peptic ulcer disease (where indicated, $p<0.05$, otherwise not significant)

\begin{tabular}{lllll}
\hline & & \multicolumn{3}{l}{ Means (SD) } \\
\cline { 3 - 5 } Groups & Characteristic & Number & Peak count (kcps) & Time (min) \\
\hline 1 & Tox+ Ulcer+ & 30 & $295 \cdot 1(128 \cdot 1)^{\star}$ & $18 \cdot 8(9 \cdot 86) \ddagger$ \\
2 & Tox+ Ulcer- & 9 & $346 \cdot 2(193 \cdot 9) \dagger$ & $21 \cdot 1(11 \cdot 2) \dagger$ \\
3 & Tox- Ulcer+ & 15 & $245 \cdot 3(139 \cdot 8)$ & $25 \cdot 6(12 \cdot 8)$ \\
4 & Tox- Ulcer- & 22 & $175 \cdot 7(116 \cdot 7)$ & $31 \cdot 1(11 \cdot 3)$
\end{tabular}

${ }^{*} \mathrm{p}<0.05$ with a $95 \% \mathrm{CI}(38.6,200.6)$ for difference in means between group 1 and $4 . \mathrm{tp}<0.05$, $95 \%$ CI $(5 \cdot 7,335 \cdot 3)$ between 2 and $4 . \neq \mathrm{p}<0.05,95 \%$ CI $(-19 \cdot 6,-5 \cdot 2)$ between 1 and 4 . p $<0.05,95 \%$ CI $(-19 \cdot 2,-0.9)$ between 2 and 4 . (Two way analysis of variance and multiple comparison).

TABLE IV Peak count and time to reach peak in the chemiluminescence response induced by 42 opsonised strains of $\mathrm{H}$ pylori in relation to the toxinogenicity and the presence or absence of peptic ulcer

\begin{tabular}{lllll}
\hline & & \multicolumn{3}{l}{ Means $(S D)$} \\
\cline { 4 - 5 } Groups & Characteristic & Number & Peak count (kcps) & Time (min) \\
\hline 1 & Tox+ Ulcer+ & 15 & $522 \cdot 1(165 \cdot 7)$ & $13 \cdot 4(3 \cdot 7)$ \\
2 & Tox + Ulcer- & 7 & $504 \cdot 3(168 \cdot 4)$ & $13 \cdot 4(3 \cdot 6)$ \\
3 & Tox- Ulcer+ & 10 & $472 \cdot 5(128 \cdot 4)$ & $13 \cdot 5(3 \cdot 8)$ \\
4 & Tox- Ulcer- & 10 & $440 \cdot 2(159 \cdot 6)$ & $14 \cdot 5(4 \cdot 7)$ \\
\hline
\end{tabular}

${ }^{\star} p>0 \cdot 05$, two way analysis of variance.

complement rather than serum antibody is the main opsonin in this respect. However, it is not certain whether complement mediated opsonisation occurs within the gastric mucosa during $H$ pylori infection. A few opsonised strains gave a decreased chemiluminescence response than non-opsonised. The reason for this is not known. There is a possibility that some serum proteins may act as electron scavengers. ${ }^{19}$

Current studies from other laboratories suggest that there are some differences between strains of $H$ pylori in their ability to produce cytotoxin and CagA protein and to cause peptic ulcer disease. ${ }^{20}$ Although host factors play a very important part in the genesis of peptic ulcer, there is increasing evidence from molecular biology and immunology for the division of $H$ pylori strains into at least two major groups one producing the CagA protein and cytotoxin (type I) and one that does not produce these antigens (type II). ${ }^{21}$ Our study here also showed there is strain to strain variation in the ability to produce cytotoxin, and to induce an oxidative burst in PMNs.

Previous studies showed that CagA/cytotoxin positive strains of $H$ pylori are associated with the infiltration of neutrophils in gastric mucosa ${ }^{1620}$ and also related to an increased expression of interleukin 8 , which is a novel cytokine capable of activating neutrophils. ${ }^{22}$ Based on these results and the findings in this study that indicate a positive association between the neutrophil respiratory burst and the cytotoxicity of $H$ pylori, which is also associated with peptic ulceration, it seems, therefore, cytotoxin/CagA positive strains may induce a more aggressive inflammatory response through secretion of toxic component(s), production of cytokines by gastric mucosal cells, recruitment of neutrophils, release of free oxygen radicals, and subsequently cause tissue damage, which may lead to peptic ulceration.
There were some strains that did not produce cytotoxin but could induce a high chemiluminescence response, raising the possibility that some $H$ pylori product(s) other than cytotoxin may be important in this respect. So far the nature of the component(s) of $H$ pylori that activates neutrophils is inconclusive. Several reports suggested that the activity is mainly caused by a protein(s), although some non-protein molecules such as lipopolysaccharides may also be implicated. ${ }^{1323}$ Whether the cytotoxin itself (or some other factor) is responsible needs further studies. There is a possibility that the gene encoding for the protein that activates PMNs is coexpressed on the so called type I strains that produce cytotoxin and CagA protein.

In conclusion, toxinogenicity of $H$ pylori strains seems to be correlated with free oxygen radical formation by neutrophils and peptic ulceration. The ability of some $H$ pylori strains to produce cytotoxin and to induce an oxidative burst of neutrophils may be important in the pathogenesis of peptic ulceration.

We thank Dr Stephanie McLaughlin for statistical analysis

This paper was presented in part at the VIIIth Internationa Workshop on Gastro-duodenal Pathology and Helicobacter pylori, 7-9 July 1995, Edinburgh.

1 Halliwell B, Gutteridge JMC, Cross CE. Free radicals antioxidants and human disease: where are we now? $f \mathrm{Lab}$ Clin Med 1992; 119: 598-620.

2 Simmonds NJ, Rampton DS. Inflammatory bowel disease adical view. Gut 1993; 34: 865-8.

3 Phull PS, Green CJ, Jacyna MR. A radical view of the stomach: the role of oxygen-derived free radicals and antioxidants in gastroduodenal disease. Eur $\mathcal{f}$ Gastroenterol Hepatol 1995; 7: 265-74.

4 Davies GR, Simmonds NJ, Stevens TRJ, Grandison A Blake DR, Rampton DS. Mucosal reactive oxygen metabolite production in duodenal ulcer disease. Gut 1992; 33: 1467-72.

5 Grisham MB, Granger DN. Neutrophil-mediated mucosal injury. Role of reactive oxygen metabolites. Dig Dis Sci 1988; 33 (suppl): 6-15S

6 Hernandez LA, Grisham MB, Twohig B, Arfors KE, Harlan JM, Granger DN. Role of neutrophils in ischaemia-reperfusion-induced microvascular injury. $\mathrm{Am}$ ischaemia-reperfusion-induced

7 Weiss SJ. Tissue destruction by neutrophils. N Engl f Med 1989; 320: 365-76.

8 Davies GR, Simmonds NJ, Stevens TRJ, Sheaff MT, Banatvala N, Laurenson IF, et al. Helicobacter pylori stimulates antral mucosal reactive oxygen metabolite production in vivo. Gut 1994; 35: 179-85.

9 Leunk RD, Johnson PT, David BC, Kraft WG, Morgan DR. Cytotoxic activity in broth-culture filtrates of Campylobacter pylori. $\mathcal{F}$ Med Microbiol 1988; 26: 93-9.

10 Eaton KA, Brooks CL, Morgan DR, Krakowka S. Essential role of urease in pathogenesis of gastritis induced by Helicobacter pylori in gnotobiotic piglets. Infect Immun 1991; 59: 2470-5.

11 Figura N, Guglielmetti P, Rossolini A, Barberi A, Cusi G, Musmanno RA, et al. Cytotoxin production by Campylobacter pylori strains isolated from patients with peptic ulcers and from patients with chronic gastritis only. f Clin Microbiol 1989; $27: 225-6$.

12 Crabtree JE, Figura N, Taylor JD, Bugnoli M, Armellini D, Tompkins DS. Expression of 120 kilodalton protein and cytotoxicity in Helicobacter pylori. $\mathcal{F}$ Clin Pathol 1992; 45: 733-4.

13 Mai UEH, Perez-Perez GI, Wahl LM, Wahl SM, Blaser MJ, Smith PA. Soluble surface proteins from Helicobacter pylori activate monocytes/macrophages by lipopolysacchride-independent mechanism. F Clin Invest 1991; 87: 894-900

14 Evans DJ, Evans DG, Takemura T, Nakano H, Lampert HC, Graham DT, et al. Characterization of a HC, Graham DT, et al. Characterization of a

15 Taha AS, Fraser WD, Kelly RW, Gemmell CG, Lee FD, Russell RI. Inhibition of human gastric cyclic AMP production by Helicobacter pylori protein - possible involvement of mucosal prostaglandin E2. Aliment Pharmacol Therap 1991; 5: 379-89.

6 Cover TL, Cao P, Lind CD, Tham KT, Blaser MJ Correlation between vacuolating cytotoxin production by Helicobacter pylori isolates in vitro and in vivo. Infect Immun 1993; 61: 5008-12. 
17 Mckinlay AW, Young A, Russell I, Gemmell CG. Opsonic requirements of Helicobacter pylori. $\mathcal{F}$ Med Microbiol 1993; 38: 209-15

18 Das SS, Karim QN, Easmon CSF. Opsonophagocytosis of Campylobacter pylori. F Med Microbiol 1988; 27: 125-30.

19 Briheim G, Stendahl O, Dahlgren C. Intra- and extracellular events in luminol-dependent chemiluminescence of
polymorphonuclear leukocytes. Infect Immun 1984; 45: polym

20 Crabtree JE, Taylor JD, Wyatt JI, Heatley RV, Shallcross TM, Tomplins DS, et al. Mucosal IgA recognition of Helicobacter pylori $120 \mathrm{KDa}$ protein, peptic ulceration and gastric pathology. Lancet 1991; 338: 332-5.
21 Xiang Z, Censini S, Bayeli PF, Telford JL, Figura N, Rappuoli $R$, et al. Analysis of expression of CagA and VacA virulence factors in 43 strains of Helicobacter pylori reveals that the clinical isolates can be divided into two major types and the CagA is not necessary for the expression of the vacuolating toxin. Infect Immun 1995; 63: 94-8.

22 Crabtree JE, Farmery SM, Lindley IID, Figura N, Peichl P, Tompkins DS. CagA cytotoxic strains of Helicobacter pylori and interleukin-8 in gastric epithelial cell lines. $\mathcal{F}$

23 Nielson $H$, Andersen LP. Activation of human phagocyte oxidative metabolism by Helicobacter pylori. Gastroenterology 1992; 103: 1747-53. 